\title{
Validated MALDI-TOF/TOF Mass Spectra for Protein Standards
}

\author{
Jayson A. Falkner, Maureen Kachman, Donna M. Veine, Angela Walker, \\ John R. Strahler, and Philip C. Andrews \\ National Resource for Proteomics and Pathway (NRPP), Michigan Proteome Consortium (MPC), Department \\ of Biological Chemistry, University of Michigan, Ann Arbor, Michigan, USA
}

A current focus of proteomics research is the establishment of acceptable confidence measures in the assignment of protein identifications in an unknown sample. Development of new algorithmic approaches would greatly benefit from a standard reference set of spectra for known proteins for the purpose of testing and training. Here we describe an openly available library of mass spectra generated on an ABI 4700 MALDI TOF/TOF from 246 known, individually purified and trypsin-digested protein samples. The initial full release of the Aurum Dataset includes gel images, peak lists, spectra, search result files, decoy database analysis files, FASTA file of protein sequences, manual curation, and summary pages describing protein coverage and peptides matched by MS/MS followed by decoy database analysis using Mascot, Sequest, and X!Tandem. The data are publicly available for use at ProteomeCommons.org. (J Am Soc Mass Spectrom 2007, 18, 850-855) (c) 2007 American Society for Mass Spectrometry

$\mathrm{T}$ Tandem mass spectrometry (MS/MS) of peptides is currently the primary method to identify proteins in complex samples. Search programs such as SEQUEST [1], Mascot [2], and X!TANDEM [3] are some of the most widely used software packages to identify the most likely peptide sequence to match an MS/MS spectrum. Development of better MS/MS identification tools is an active area of proteomics research [4-7], MS/MS de novo tools [8-10], MS/MS spectral search tools [11, 12], and MS/MS search result refinement tools $[13,14]$. All of these tools rely on libraries of well-studied MS/MS spectra from a variety of instruments with accurate peptide assignments.

Accurate peptide assignments are essential but manual confirmation is a time-consuming process, subject to some degree of operator subjectivity, and is not feasible for high-throughput proteomic analysis. Most commonly, MS/MS algorithms are trained on in-house generated datasets that have undergone a variety of selection criteria to verify their authenticity. These standard sets are often obtained from analysis of commercial protein preparations with limited criteria for purity or represent bootstrap efforts that set stringent criteria for results from existing search engines. Recently, several approaches have been proposed to accurately estimate falsepositives and associate peptide identifications with MS/MS spectra with high levels of confidence [13,

Published online February 27, 2007

Address reprint requests to Dr. Philip C. Andrews, University of Michigan, Biological Chemistry, 300 N. Ingalls 11th Fl. 1198, Ann Arbor, MI 48109. E-mail: andrewsp@umich.edu
15]. Development of MS/MS-related algorithms and tools would greatly benefit from publication of thirdparty datasets, particularly well-annotated datasets using these proposed approaches to estimating falsepositives with as much manual confirmation as possible. Finally, the availability of well-verified sets of MS/MS spectra can provide the basis for direct spectral comparison, which has the potential to be a much more effective approach to peptide identification that existing engines that match against generated MS/MS spectra and obviates the need for an accurate fragmentation model.

Small reference sets of tryptic peptides have been made from known proteins $[16,17]$ and larger datasets have been made from the yeast proteome $[18,19]$ and human serum proteins collectively in the HUPO initiative [20]. Although these are useful databases, they are time consuming to generate and are not publicly available as a reference set.

In this manuscript we describe a publicly available library of tandem mass spectra generated on an ABI 4700 MALDI TOF/TOF from 246 known purified and trypsin-digested protein samples using a workflow used for gel-purified proteins. The data are analyzed using the Mascot, X!Tandem, and Sequest search engines, and peptide identifications are adjusted to $99 \%$ true-positive confidence using the intuitive decoy database approach described by Elias et al. [15]. In addition to the peak lists and associated peptide identifications, the described dataset is also published with the raw spectra, search result files, decoy database analysis files, Scaffold analysis files, and the gel images used when checking for protein purity. 


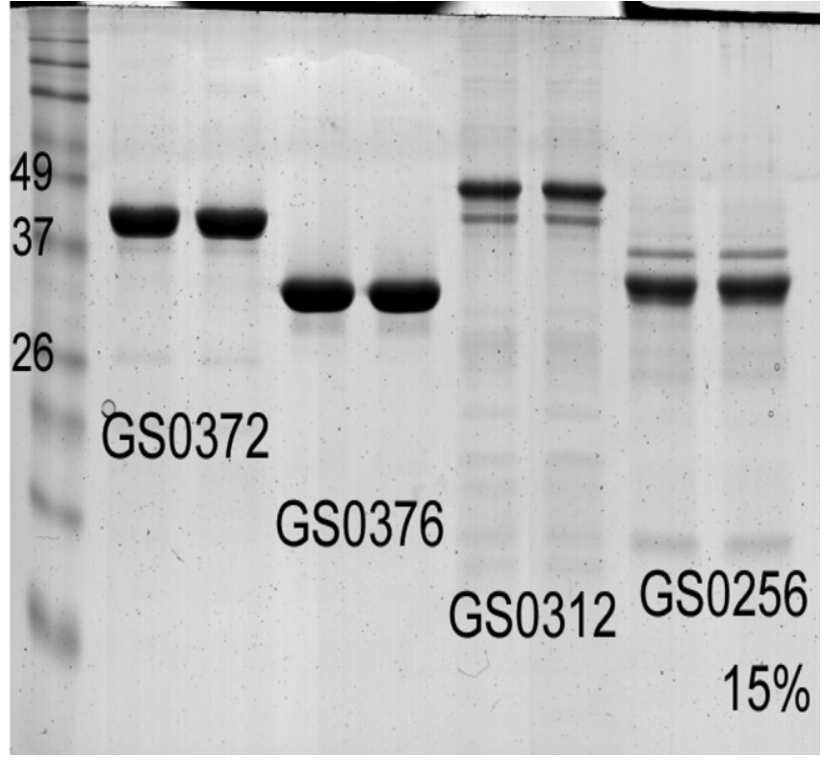

Figure 1. Example SDS-PAGE check for protein purity. Each protein in the Aurum Dataset was checked for purity using a hand-cast polyacrylamide gel run under protein denaturing conditions (SDS-PAGE). Multiple proteins were run on the same gel and each protein was run in two lanes. Proteins are labeled by their Aurum identification number. Only the predominant band was excised, digested, and analyzed using MS/MS. A gel image is included with this manuscript's data for every protein analyzed and the gels are linked in the protein summary report (see Figure 2). Gel images also include a protein standard ladder in the first lane (approximate $\mathrm{kDa}$ labeled) and the percentage polyacrylamide used to cast the gel in the bottom right.

\section{Experimental}

\section{Proteins}

A selection of 300 sequence-verified recombinant human proteins $(8-70 \mathrm{kDa})$ were obtained from GenWay Biotech (San Diego, CA). The proteins were purchased from GenWay Biotech representing existing clones that could be readily expressed and purified. GenWay Biotech sequence-verified the clones and a report for each cloned sequence is included in GenWay's product documentation, which is referenced by the per protein report included in the on-line Aurum documentation. After purity analyses, 246 of the 300 proteins were used in the Aurum analysis. The proteins contained an N-terminal T7 tag (MASMTGGQQMG also observed as ASMTGGQQR) or His6 tag (HHHHHH) and were expressed in Escherichia coli. Documentation provided with the proteins included the name, expressed length, and the NCBI accession number. Proteins $(2 \mu \mathrm{g} /$ lane $)$ were analyzed for purity by SDS-PAGE stained with colloidal Coomassie G-250 (Figure 1). The criteria for purity were that at least $50 \%$ of the protein was at the correct size, the gel lane contained no nearby unrelated protein, and at least $95 \%$ of the tryptic peptides corresponded to the anticipated protein. Images for each of the gels are included in the supplementary data and shown directly on individual protein summary pages (Figure 2).
For each protein in the Aurum dataset a unique GS-number was assigned where we used the letters "GS" followed by four digits representing a decimal number assigned to the protein. The supplementary data includes a table that maps this GS number to an appropriate GI number, NCBI accession number, and Swissprot acession number. The GS nomenclature is not intended to represent a new standard for referring to the associated protein sequence. Rather it is a convenient way to uniquely identify Aurum proteins for internal use within the dataset-independent of accession number or identifier changes that might occur in other databases. GS numbers will remain static throughout the lifespan of the Aurum dataset; however, use of the GS numbers outside the context of analyzing the Aurum data is discouraged when either NCBI or Swissprot identifiers are available.

\section{Protein Coverage Calculations}

The entire protein sequence is included in each summary file as shown in one file in Figure 2. The sequence is further colored to indicate the peptides that were identified and the portions of protein sequence that are not expected to be identified. The portions that are not expected to be identified are those that have $\mathrm{m} / \mathrm{z}$ at +1 charge of $<900 \mathrm{Da}$ or $>2500 \mathrm{Da}$; that is, the range that the mass spectrometer is configured to ignore. Data analysis for this manuscript is based on a MALDI instrument, and the +1 charge state is almost ubiquitously observed for ionized peptides. Thus the theoretical $\mathrm{m} / \mathrm{z}$ of an ionized peptide is well approximated to be its molecular mass. The range of 900 to $2500 \mathrm{Da}$ is selected for three primary reasons. First, MALDI instruments are prone to ionizing matrix clusters that can dominate the lower mass region, which often makes it very difficult to identify anything below the mass of 900 $\mathrm{Da}$. Second, the instrument used for this analysis is tuned to most accurately identify ions with $\mathrm{m} / \mathrm{z}$ of 1800 Da. Ions with much less or much greater $\mathrm{m} / \mathrm{z}$ may report an incorrect $\mathrm{m} / \mathrm{z}$ to the point where it is difficult to use in data analysis. Third, the MALDI TOF/TOF does not detect higher mass peptides as well as lower and the trade-off between higher $\mathrm{m} / \mathrm{z}$ and the amount of sample required to detect the ion appears to be nonlinear. Thus, peptides with masses $>2500$ are problematic because of both relatively inaccurate $\mathrm{m} / \mathrm{z}$ measurements and relatively poor signal strength.

Explanation of protein coverage is important because the results section and included protein reports present two types of protein coverage information. The first type of protein coverage is a strict percentage of the total protein sequence covered by observed peptides. The second type of coverage- "expected protein coverage" - is the percentage of tryptic peptides that fall within the 900 to $2500 \mathrm{Da}$ range (i.e., the peptides one might expect to see based on the Aurum data acquisition parameters). 
(a)

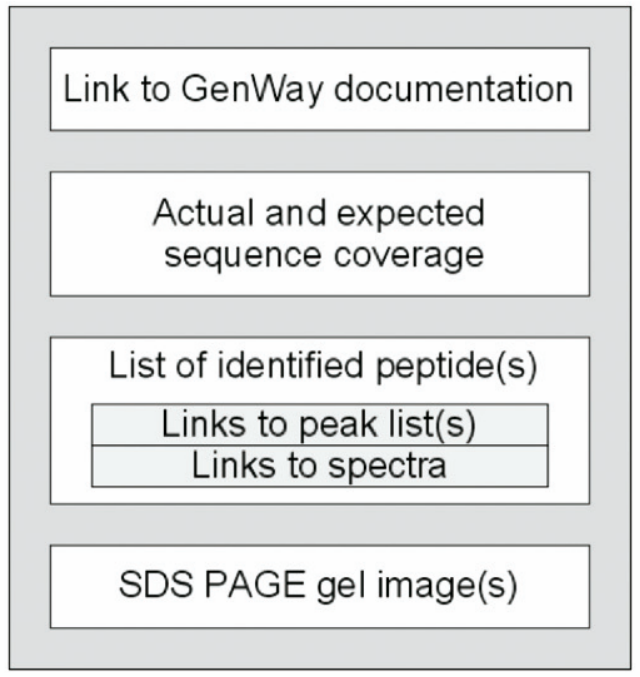

(b) Protein Coverage

This is the protein coverage report for >GS0119|6598323|NP_001485|P50: MS ions to further fragment. Green sections highlight peptides that were fou

Peptides that could have been observed

Peptides that shouldn't be observed (m/z less than 900 Da or more th Peptides that were observed.

NEE YDVIVLGTGL TEC ILSG I MSVNGKKVL HMDRNPYYGGES AS ITPLEDL YKRF KIPGSPPESI

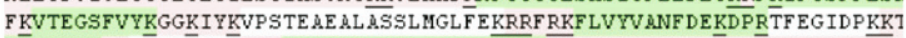
CYETINR IKL YSESLARYGKSPYL YPL Y GL GELPQGF ARLS A I Y G GTYMLNKP IEE I IVQNGKV I CILSHP IKNTND ANSCQI I I PQNQVNRKSD I YVCM ISF AHNV A A QGKY I A IVST TVE TKEPEKE I RTYDATTHFETTCD D I KNN I YKRMTGSEF DFEEMKRKKND I YGED

\section{Statistics/Information}

- Total Protein Coverage: 215 out of 444 residues or $48.42 \%$ - Expected Protein Coverage: 215 out of 310 residues or $69.35 \%$

Figure 2. Protein report file structure and partial example. (a) Block diagram describing the information in each of the protein report files included with the supplementary information. The intention of these pages is to provide a human-friendly summary for each protein analyzed. (b) Example protein coverage information from a protein summary page. This is only a portion of the summary page highlighting total protein coverage and coverage of expected peptides. Not shown in the figure are the complete statistics, peptide matches, and links to spectra, peak lists, and other documentation.

\section{In-Gel Tryptic Digestion}

Excised gel plugs $(0.67 \mu \mathrm{g}$ protein) were placed in 96-well plates and were processed using a MassPrep robotic workstation (Waters, Milford, MA, USA). The plugs in the presence of $50 \mathrm{mM}$ ammonium bicarbonate underwent the following steps: wash/dehydration with $50 \%$ acetonitrile; reduction with $10 \mathrm{mM}$ DTT; alkylation with $55 \mathrm{mM}$ iodoacetamide; wash/dehydration with $50 \%$ acetonitrile; digestion for $4 \mathrm{~h}$ with trypsin (200 ng, porcine, modified, Promega, Madison, WI, USA). Peptides were extracted from the gel plug with $1 \%$ formic acid $/ 2 \%$ acetonitrile and concentrated using C-18 ZipTips (Millipore, Bedford, MA, USA). Digests were spotted (four replicates) on a MALDI target using $\alpha$-cyano 4-hydroxy cinnamic acid $(2 \mathrm{mg} / \mathrm{ml}$ in $50 \%$ acetonitrile, $0.1 \%$ TFA containing $10 \mathrm{mM}$ ammonium phosphate) as matrix. Dilutions of the digests were made at $1 / 8$ and were spotted in the same manner.

\section{MS/MS Acquisition}

Spectra were acquired on a 4700 MALDI TOF/TOF mass spectrometer (Applied Biosystems, Foster City, CA, USA). Spectra were acquired for the eight most intense ions. In a replicate well, after excluding the seven most intense ions, the next eight most intense ions were analyzed. Similarly, the next set of eight ions was analyzed for wells 3 and 4 . Known trypsin autodigestion peptides were excluded. This process resulted in acquisition of a maximum of 32 spectra per digest, theoretically 29 unique spectra if sample and MS intensities do not change between spottings.

\section{Data Curation}

Default peak lists from Applied Biosystems GPS software were taken from replicate wells and were concatenated into a single Mascot Generic Format (.mgf) file. To map spectra back to the original files the the base 16 encoding of individual file's MD5 [22] hash was set as the MGF file's TITLE field. Additionally, all peak lists were converted into a set of .dta files by the ProteomeCommons.org IO Framework [21] for subsequent analysis by the Sequest. Four different initial searches were performed, each using $0.5 \mathrm{Da}$ for the parent and fragment ion mass accuracy and with oxidation $(\mathrm{M}, \mathrm{H}, \mathrm{W})$, deamidation $(\mathrm{N}, \mathrm{Q})$ variable modifications. Iodoacetamide (C) was specified as a static modification. Four follow-up searches were performed using the similar parameters but without iodoacetamide as a static modification in favor of setting iodoacetamide and propionamide as variable modifications for the side chains of cysteine residues. Three of the four searches used different search engines in an attempt to identify as many of the spectra as possible. The two same-search engine searches both used Sequest but included the variable $n$-term protein modifications for each of the two purification tags.

The MS/MS searches were performed using Mascot, X!Tandem, and Sequest. Two of the initial searches used the concatenated .mgf file. One search on Mascot 1.9 and the other search on X!Tandem 06_9_15. X!Tandem did by default include N-term pyro-glu from $\mathrm{N}$ and $\mathrm{Q}$ as modifications. The other two initial searches used Sequest. One search assuming each protein had an N-term T7 tag and the other 
search assuming each protein had a N-term HIS tag. All searches were performed on a decoy database version of the IPI Human FASTA file version 3.14. The decoy database was the exact IPI Human 3.14 FASTA file with a concatenated reverse version of the same database. Each protein in the reverse sequence is noted by appending an " $R$ " to the protein's accession number and each protein is changed by reversing the order of the amino acid residues. The ProteomeCommons.org IO Framework was used to generate the reverse database [21].

Identification of peaklists was based on the decoy search strategy outlined by Elias et al. [15] and described briefly here. Each search engine's peptide identifications were individually ranked according to the respective following scores: Mascot's ion score, X!Tandem's hyperscore, and Sequest's XCorr. Each sorted list is then filtered to include only matches that scored above a 99\% confidence threshold determined as follows. All peptides above the score are binned into two categories. Those that are from the normal FASTA sequences (i.e., matches without a " $\mathrm{R}$ " in the accession) and those that are from the decoy sequences (i.e., matches with a " $R$ " in the accession). The false-positive rate is estimated to be twice as much as the ratio of decoy sequences versus normal sequences-twice because the decoy sequences represent only half the total database-thus approximately as many normal sequences are likely inaccurate. An example would be the case where 198 normal sequences were identified per every 1 decoy sequence, where $(1 \times 2) / 200$ yields a $1 \%$ false-positive rate, equivalent to $99 \%$ confidence in individual peptide identifications. This strategy is presented as an appropriate objective analysis of the dataset that takes advantage of individual expertise present in different search engines while still normalizing all search results to roughly $99 \%$ confidence in true positives.

Files used by the respective search engines, including search parameter files, peak list files, and FASTA files, are included with the on-line download as described below in the availability section.

\section{Results and Discussion}

A well-documented set of purified human recombinant proteins has been procured and analyzed using a routine gel-based protocol to generate a library of mass spectra referred to as the Aurum dataset. Now the Aurum dataset consists of 246 recombinant human proteins that have been trypsin-digested and characterized by MALDI TOF/TOF. The MS/MS dataset further underwent what is intended to be an objective, community-standard-based analysis to generate spectra-associated peptide identifications and protein coverage information.

The recombinant proteins were expressed in E. coli and initial isolation performed by the vendor. Upon receipt, the proteins were analyzed by SDS-PAGE for purity and the dominant bands were excised for in-gel tryptic digestion. Figure 1 shows a representative gel, where samples GS0372 and GS0376 represent the highest purity provided and samples GS0312 and GS0256 represent a moderate to low purity. Of the 246 proteins, 173 were represented as a single band and were classified as high purity. An additional 17 were represented as evenly distributed doublets. Analysis of both bands of the doublets confirmed that both were forms of the target protein and could be placed in the subset of high-purity proteins that would be suitable for future in-solution digests. The remaining proteins had varying degrees of contaminating bands ranging from possible truncation products to E. coli proteins. Only the predominant band was excised and characterized. Only proteins for which all tryptic peptides returned the correct ID were included in the final protein list. Gel images are included for each protein in the protein's summary page found with the on-line documentation for the Aurum Dataset.

MS/MS analysis for the selected gel bands was carried out according to the standard analysis procedures described in the experimental section. Seven different MALDI plates were used, found in the documentation with the names "T10467," "T10475," “T10622," “T10645," “T10707," “T10739," and “T10761." Each protein was spotted individually at least four times to help ensure the best chance of acquiring high-quality spectra for as many of the peptides associated with each protein as possible. At least 32 spectra were acquired for each protein by collecting data from four separate spots of the protein as described in the experimental section. Default peak lists of the spectra were then extracted for analysis using the MSExtractor tool (http://www.proteomecommons.org/current/489) and concatenated using the ProteomeCommons. org IO Framework [21]. In all 9987 total peaklists are included in the resulting .mgf file. Each peak list is identified by the original file's MD5 hash listed in the TITLE field of the associated peak list in the .mgf file.

Decoy database analysis targeting 99\% true-positive confidence and using Mascot, X!Tandem, and Sequest were performed according to suggested guidelines published by Elias et al. [15]. The analysis is not intended to be a comparison of the search engines used; rather it normalizes the results of each search engine to an approximated $99 \%$ true-positive confidence. All of the peptides from the $99 \%$ true positive results were aggregated to make the set of all identified spectra. In all 5054 unique peptide sequences ( $>50 \%$ of all peak lists) were identified at $99 \%$ true-positive confidence with the peptides being identified coming from the initial and follow-up searches described in the following format (initial search)/(follow-up search). Note that the followup search is not intended to identify a superset of peptides and the notation does not indicate a fraction. Mascot identified 1682/1847 peptides, X!Tandem identified 441/424 peptides, and Sequest identified 2939/ 2921 peptides for the T7 tag and 2937/2920 peptides for 
the HIS tag searches. No search engine identified a superset of all others and a significant gain in highly confident identifications was obtained by combining results from all three search engines. These results appear to support the use of decoy database analysis with multiple search engines as an approach to identify more spectra from a dataset; again, however, it is worth emphasizing that these results are not intended as a comprehensive basis for comparison of the search engines used. Various search results are expected because each search engine performs analysis differently even with the similar settings used in each search. Additionally, each search engine has a disparate range of settings that might be optimized to change the results of the analysis. The set of search engines used is intended only to help increase the number of unique spectra identified. For further analysis, the same search result files used for decoy database analysis were imported into the Scaffold software package (ProteomeSoftware Inc., Portland, OR, USA) for PeptideProphet and ProteinProphet-like analysis. Similar results as for the decoy database analysis were found and the free Scaffold Viewer program may be used to examine the Scaffold files included with this manuscript.

Of the 246 purified proteins 242 proteins had at least one peptide identified to the expected cloned sequence and 233 peptides had more than two peptides matched to the expected cloned sequence. At the most, up to 19 unique peptides matched to a cloned protein. The average protein sequence coverage from this analysis was $32 \%$ and the average protein coverage of theoretically detectable peptides was $63 \%$. Theoretically detectable peptides include those that have an unmodified $\mathrm{m} / z$ of $>900 \mathrm{Da}$ and $<2500 \mathrm{Da}(\mathrm{m} / z$ restrictions specified at the time of data collection). A summary of protein coverage and matched peak lists are provided for each protein in the supplementary files described below in the availability section. Figure 2 shows an example protein summary. Analysis of the summary files illustrates that the majority of proteins have several peptides that may be used to identify them from a biological sample assuming that similar quantities of the purified protein and/or peptides can be obtained, although some of the proteins have very few, if any, peptides that are readily observed. These proteins are of interest for further study because they may represent proteins that are difficult to identify from a potentially more complex sample using a similar MALDI TOF/ TOF-based approach. A simple explanation for several of these difficult to analyze proteins is that they are relative small proteins with very few, if any, tryptic peptides that fall within the 900-2500 Da cutoff used when analyzing this dataset. Potentially a different mass spectrometer such as an electrospray ionization (ESI)-based instrument or a different digestion protease would provide a more favorable analysis. Other plausible explanations could account for the difficulty in analysis of other proteins such as poor ionization of the peptides, limited fragmentation, unexpected modi- fications, unfavorable experimental protocols for analyzing the particular protein, or even experimental error. In any case, the set of poorly identified proteins may be of interest for further analysis to identify whether they are indeed poor candidate proteins for mass spectrometry analysis.

Further data analysis and summary reports were generated to check for common contaminants in mass spectrometry experiments. The cRAP (pronounced "cee-RAP") 1.0 list of proteins maintained at TheGPM. org was searched against the unidentified peak lists. The cRAP list contains about 100 proteins including common laboratory proteins, proteins added by accident through dust or physical contact, and proteins commonly used as molecular weight standards. The cRAP analysis was performed using just the X!Tandem search engine and 28 proteins were found with more than two peptides matching. In all, 37 proteins were identified by one or more peptides. The proteins primarily identified included many keratin proteins and several E. coli proteins. BSA and Serotransferrin were also found. The complete list of cRAP proteins, the $\mathrm{X}$ !Tandem search results, and a set of summary pages similar to Figure 2 for the cRAP proteins are included with the supplementary data.

\section{Conclusions}

The Aurum Dataset is a high-quality dataset of known proteins analyzed by a MALDI TOF/TOF. The proteins are all human proteins expressed in E. coli and purified by N-terminus T7 and HIS tags. The proteins further purified using SDS-PAGE, individually digested with trypsin, and individually spotted four times on a MALDI plate. Data were acquired to represent at least the top 29 most intense MS peaks, and published decoy database analysis was used to identify more than $50 \%$ of the acquired spectra: roughly 5000 unique peptides. Based on this analysis, the majority of proteins can readily be identified, although a range exists where some proteins are not as easily analyzed. The low end of this range is of particular interest for further analysis because it might be helpful to identify why certain proteins are more difficult to identify from complex samples.

The Aurum Dataset is a valuable contribution for testing existing MS/MS algorithms and tools, and the Aurum Dataset will be helpful as an objective thirdparty dataset for developing new tools and algorithms. The published dataset contains all raw and curated data used to generate the analysis described by this manuscript and all these data are openly and freely available for use (see Appendix below).

\section{Appendix}

The Aurum Dataset is freely available for use in its entirety from ProteomeCommons.org. On-line versions of the data may be found at http://www.proteome 
commons.org/current/553/index.html or by searching on the keyword "Aurum" from the proteomecommons. org main page.

The ProteomeCommons.org Tranche network is used to provide fast downloads of the data and to get a verifiable, exact copy of the data described by this manuscript. The Tranche hash for the Aurum Dataset is given below:

HnxUzQuuP7BIqF10aetLtjwnffOwuOM-AfDvg2BF menNe9UeMgprBFh7+wtpbcWnXqMk2KY-8z9Vjmw qXYDbQ0pTNqIx4AAAAAASJlaw $==$

Further information about Tranche and how to use this hash may be found on-line at http://www. proteomecommons.org/dev/dfs/ (see Appendix below).

\section{Acknowledgments}

The authors thank Mary Hurley, Sara Volk, and Jennifer Callahan from the Michigan Proteome Consortium (MPC) for expert technical assistance and Tom Blackwell and Pete Ulintz for helpful discussions. The authors also thank Anastasia Yocum for assistance in the Sequest database searches and work with Scaffold. This work was supported by NIH/NCRR, National Resource for Proteomics and Pathway Mapping Grant P41-18627, and State of Michigan MEDC Grant GR239.

\section{References}

1. Eng, J. K.; McCormack, A. L.; Yates, J. R., III. An Approach to Correlate Tandem Mass Spectral Data of Peptides with Amino Acid Sequences in a Protein Database. J. Am. Soc. Mass Spectrom. 1994, 5, 976-989.

2. Perkins, D. N.; Pappin, D. J.; Creasy, D. M.; Cottrell, J. S. Probabilitybased Protein Identification by Searching Sequence Databases Using Mass Spectrometry Data. Electrophoresis 1999, 20, 3551-3567.

3. Craig, R.; Beavis, R. C. TANDEM: Matching Proteins with Mass Spectra. Bioinformatics 2004, 20, 1466-1467.

4. Clauser, K. R.; Baker, P. R.; Burlingame, A. L. Role of Accurate Mass Measurement $( \pm 10 \mathrm{ppm})$ in Protein Identification Strategies Employing MS or MS/MS and Database Searching. Anal. Chem. 1999, 71, 2871-2882.

5. Narasimhan, C.; Tabb, D. L.; Verberkmoes, N. C.; Thompson, M. R.; Hettich, R. L.; Uberbacher, E. C. MASPIC: Intensity-based Tandem Mass
Spectrometry Scoring Scheme That Improves Peptide Identification at High Confidence. Anal. Chem. 2005, 77, 7581-7593.

6. Colinge, J.; Masselot, A.; Cusin, I.; Mahe, E.; Niknejad, A.; Argoud-Puy, G.; Reffas, S.; Bederr, N.; Gleizes, A.; Rey, P.; Bougueleret, L. Highperformance Peptide Identification by Tandem Mass Spectrometry Allows Reliable Automatic Data Processing in Proteomics. Proteomics 2004, 4, 1977-1984.

7. Tabb, D. L.; Narasimham, C.; Strader, M. B.; Hettich, R. L. DBDigger: Reorganized Proteomic Database Identification That Improves Flexibility and Speed. Anal. Chem. 2005, 77, 2464-2474.

8. Taylor, J. A.; Johnson, R. S. Implementation and Uses of Automated de Novo Peptide Sequencing by Tandem Mass Spectrometry. Anal. Chem. 2001, 73, 2594-2604.

9. Ma, B.; Zhang, K.; Hendrie, C.; Liang, C.; Li, M.; Doherty-Kirby, A.; Lajoie, G. PEAKS: Powerful Software for Peptide de Novo Sequencing by Tandem Mass Spectrometry. Rapid Commun. Mass Spectrom. 2003, 17, 2337-2342.

10. Frank, A.; Pevzner, P. PepNovo: De Novo Peptide Sequencing via Probabilistic Network Modeling. Anal. Chem. 2005, 77, 964-973.

11. Craig, R.; Cortens, J. C.; Fenyo, D.; and Beavis, R. C.; "Using Annotated Peptide Mass Spectrum Libraries for Protein Identification. J. Proteome Res. 2006, 5, 1843-1849.

12. National Institute of Standards and Technology (NIST). NIST Library of Peptide Ion Fragmentation Spectra: Version June 2006, http:// chemdata.nist.gov/mass-spc/ftp/mass-spc/PepLib.pdf

13. Keller, A.; Nesvizhskii, A. I.; Kolker, E.; Aebersold, R. Empirical Statistical Model to Estimate the Accuracy of Peptide Identifications Made by MS/MS and Database Search. Anal. Chem. 2002A, 74, 53835392.

14. Nesvizhskii, A. I.; Keller, A.; Kolker, E.; Aebersold, R. A Statistical Model for Identifying Proteins by Tandem Mass Spectrometry. Anal. Chem. 2003, 75, 4646-4658.

15. Elias, J. E.; Haas, W.; Faherty, B. K.; Gygi, S. P. Comparative Evaluation of Mass Spectrometry Platforms Used in Large-scale Proteomics Investigations. Nat. Methods 2005, 2, 667-675.

16. Purvine, S.; Kolker, N.; Kolker, E. Spectral Quality Assessment for High-throughput Tandem Mass Spectrometry Proteomics. OMICS 2004, $8,255-265$

17. Keller, A.; Purvine, S.; Nesvizhskii, A. I.; Stolyar, S.; Goodlett, D. R.; Kolker, E. Experimental Protein Mixture for Validating Tandem Mass Spectral Analysis. OMICS 2002B, 6, 207-212.

18. Martin, D. B.; Eng, J. K.; Nesvizhskii, A. I.; Gemmill, A.; Aebersold, R. Investigation of Neutral Loss during Collision-induced Dissociation of Peptide Ions. Anal. Chem. 2005, 77, 4870-4882.

19. Tabb, D. L.; Smith, L. L.; Breci, L. A.; Wysocki, V. H.; Lin, D.; Yates, J. R. III. Statistical Characterization of Ion Trap Tandem Mass Spectra from Doubly Charged Tryptic Peptides. Anal. Chem. 2003, 75, 1155-1163.

20. Kapp, E. A.; Schutz, F.; Connolly, L. M.; Chakel, J. A.; Meza, J. E.; Miller, C. A.; Fenyo, D.; Eng, J. K.; Adkins, J. N.; Omenn, G. S.; Simpson, R. J. An Evaluation, Comparison, and Accurate Benchmarking of Several Publicly Available MS/MS Search Algorithms: Sensitivity and Specificity Analysis. Proteomics 2005, 5, 3475-3490.

21. Falkner, J. A.; Falkner, J. W.; Andrews, P. C. ProteomeCommons.org IO Framework: Reading and Writing Multiple Proteomics Data Formats. Bioinformatics 2007, 23, 262-263.

22. Rivest, R. The MD5 Message-Digest Algorithm, Network Working Group, Request for Comments. 1321, April 1992. 